\title{
Costovertebral Joint
}

National Cancer Institute

\section{Source}

National Cancer Institute. Costovertebral Joint. NCI Thesaurus. Code C32394.

The synovial joints that attach the heads of the ribs to the bodies of the thoracic vertebrae. 